\title{
ESTANDARIZACIÓN DEL DIAGNÓSTICO Y PLAN DE CUIDADOS ENFERMERO ANTE EL "RIESGO DEL SÍNDROME DE LA FRAGILIDAD DEL ANCIANO"
}

\author{
STANDARDIZATION OF THE DIAGNOSIS AND NURSING \\ CARE PLAN FOR THE RISK OF FRAIL ELDERLY SYNDROME IN OLDER \\ ADULTS
}

Carlota de Miguel Barbero

Enfermera en Centros de Atención Primaria de Andorra

Barbero, C. (2020). ESTANDARIZACIÓN DEL DIAGNÓSTICO Y PLAN DE CUIDADOS ENFERMERO ANTE EL "Riesgo del síndrome de la fragilidad del anciano". Revista Ene De Enfermería, 14(2). Consultado de http://ene-enfermeria.org/ojs/index.php/ $\mathrm{ENE} /$ article/view/980 


\section{Resumen}

La estandarización de los procesos enfermeros es uno de los retos de los diferentes sistemas sanitarios a escala mundial así como el seguimiento de la pre-fragilidad. Actualmente, el modelo de estandarización de los planes de cuidados en Atención Primaria de Andorra no es suficiente y requiere la introducción de las nuevas tecnologías para hacerlo más eficaz. Así mismo, la pre-fragilidad necesita un seguimiento de Atención Primaria. Por ello, esta investigación pretende estandarizar el plan de cuidados de los pacientes potencialmente frágiles usando la taxonomía de la NANDA-I y el estándar semántico SNOMED CT empleando la metodología Delphi en tiempo real.

Palabras clave: enfermería, fragilidad, taxonomía, NANDA-I, SNOMED CT

\section{Abstract}

The standardization of nursing processes is one of the challenges of different health systems worldwide, as well as the monitoring of pre-frailty. Actually, the model of standardization of care plans in Primary Care of Andorra isn't enough and requires the introduction of new technologies to make it more efficient and effective. Likewise, pre-frailty needs a follow-up of Primary Care specialists. Therefore, this research aims to standardize the care plan of potentially fragile patients using the NANDA-I taxonomy and the SNOMED CT semantic standard employing the Delphi methodology in real time.

Key words: Nursing, frailty, taxonomy, NANDA-I, SNOMED CT 


\section{INTRODUCCIÓN}

La fragilidad es un síndrome geriátrico que se caracteriza por la disminución de la capacidad del organismo para responder a los factores estresantes externos provocando en el individuo: riesgo de caídas, declive funcional, discapacidad, dependencia, institucionalización e incluso la muerte. En consecuencia, tiene un impacto importante en la funcionalidad y calidad de vida de las personas y en el consumo de recursos sanitarios y sociales. Por ello, se considera un verdadero problema de Salud Pública que debe ser atendido con urgencia (1-2).

Fried y otros autores, en el año 2001, plantearon una definición de un fenotipo de fragilidad de acuerdo a los siguientes cinco criterios: pérdida de peso, cansancio, baja actividad física, velocidad de la marcha ralentizada y escasa fuerza muscular; considerando que una persona está en una condición de pre-frágil si cumple con uno o dos de estos criterios y está en una condición de frágil si cumple tres o más (3).

La fragilidad está relacionada con más condiciones clínicas de las citadas y es por este motivo que el abordaje tiene como objetivo poder cribar aquellas personas vulnerables, a fin de prevenir o retrasar la aparición de consecuencias adversas que acelerarán el deterioro funcional de la persona. En Francia, Toulouse, se está trabajando en el desarrollo de la plataforma Gérontopôle para el referido cribado. Esta entidad, en 2012, creó "la Gérontopôle Frailty Screening Tool "(GFST) para identificar a la población frágil. Fue diseñada con los criterios de Fried incluyendo dos factores más que el equipo de creación de la GFST consideró también importantes en la prevención de la discapacidad; la alteración cognitiva y vivir solo (2-4).

La Organización Mundial de la Salud (OMS), recientemente ha introducido dos conceptos nuevos de evaluación exhaustiva sobre el envejecimiento: La Capacidad Intrínseca (Cl) y la Capacidad Funcional (CF). Define la $\mathrm{Cl}$ como "la composición de todas las capacidades mentales y físicas que una persona puede tener, incluyendo su capacidad para caminar, pensar, ver, escuchar y recordar estando influenciados por varios factores como la presencia de enfermedad, lesiones y cambios relacionados con la edad " y la CF como "la composición de la Capacidad Intrínseca del individuo, de las características ambientales relevantes y de la interacción entre ellas".

Acerca de las características ambientales, se incluyen los entornos como el hogar, la comunidad y la sociedad (el entorno construido, sus personas y sus 
relaciones, actitudes, valores y las políticas de salud y sociales), ya que vivir en un entorno que favorece la conservación de la $\mathrm{Cl}$ y CF es clave para un Envejecimiento Saludable definido como "proceso de desarrollo y mantenimiento de la capacidad funcional que permite el bienestar en la vejez".

Estas definiciones están incluidas en el programa ICOPE (Implementation of Integrated Care For Older People) que se centra en un enfoque integrado de la persona para mantener las $\mathrm{Cl}$ a lo largo del curso de la vida para conservar al máximo la CF, donde la intervención precoz es esencial para no convertirse en personas dependientes dentro del marco del Envejecimiento Saludable.

Las directrices del programa ICOPE se estructuran en el módulo I: Disminución de las capacidades físicas y mentales, módulo II: Síndromes geriátricos asociados con la dependencia de la atención al envejecimiento y el módulo III: Apoyo al cuidador (ver tabla 1) (5).

Tabla 1. Las Capacidades Intrínsecas y Funcionales del programa ICOPE: recomendaciones, grado de recomendación e intervenciones.

\begin{tabular}{|c|c|c|c|}
\hline $\begin{array}{l}\text { Capacidades Funcionales (CF) } \\
\text { y Capacidades Intrínsecas (CI) }\end{array}$ & Recomendaciones & $\begin{array}{l}\text { Grado de } \\
\text { recomendación }\end{array}$ & Intervenciones \\
\hline \multirow{18}{*}{$\begin{array}{l}\text { Módulo I: } \\
\text { Disminución de las capacidades } \\
\text { físicas y mentales }\end{array}$} & \multirow{3}{*}{ Pérdida de movilidad } & \multirow{3}{*}{ Fuerte } & Entrenamiento de la resistencia progresiva \\
\hline & & & $\begin{array}{l}\text { Ejercicio físico (entrenamiento de equilibrio o } \\
\text { multicomponente) }\end{array}$ \\
\hline & & & $\begin{array}{l}\text { Rehabilitación física (adaptada a las necesidades de la } \\
\text { persona mayor) }\end{array}$ \\
\hline & \multirow{3}{*}{ Malnutrición } & \multirow{3}{*}{ Fuerte } & Suplemento de nutrición oral (macro y micronutrientes) \\
\hline & & & Consejo dietético \\
\hline & & & $\begin{array}{l}\text { Estrategia a la hora de comer para mejorar la ingesta de } \\
\text { alimentos }\end{array}$ \\
\hline & \multirow[t]{2}{*}{ Discapacidad visual } & \multirow[t]{2}{*}{ Fuerte } & $\begin{array}{l}\text { Examen de detección de la deficiencia visual en los } \\
\text { entornos de atención primaria (error de refracción o } \\
\text { catarata) }\end{array}$ \\
\hline & & & $\begin{array}{l}\text { Prestación oportuna de atención oftalmológica (error de } \\
\text { refracción o catarata) }\end{array}$ \\
\hline & \multirow[t]{2}{*}{ Pérdida auditiva } & \multirow[t]{2}{*}{ Fuerte } & $\begin{array}{l}\text { Detección y provisión de audífonos o dispositivos de } \\
\text { asistencia auditiva }\end{array}$ \\
\hline & & & $\begin{array}{l}\text { Intervención pedagógica para mejorar la aceptación del } \\
\text { uso del audífono }\end{array}$ \\
\hline & \multirow{3}{*}{ Deterioro cognitivo } & \multirow{3}{*}{ Condicional } & Estimulación cognitiva \\
\hline & & & Entrenamiento cognitivo \\
\hline & & & Rehabilitación cognitiva \\
\hline & \multirow{5}{*}{ Síntomas depresivos } & \multirow{5}{*}{ Condicional } & actividad conductual \\
\hline & & & Terapia cognitiva conductual, \\
\hline & & & Terapia psicoeducativa \\
\hline & & & Terapia interpersonal \\
\hline & & & Terapia de resolución de problemas \\
\hline
\end{tabular}




\begin{tabular}{|c|c|c|c|}
\hline & & & Terapia de protocolo de atención escalonada \\
\hline & & & Terapia de revisión de vida \\
\hline \multirow{15}{*}{$\begin{array}{l}\text { Módulo II: Síndromes geriátricos } \\
\text { asociados a la dependencia de la } \\
\text { atención al envejecimiento }\end{array}$} & \multirow{5}{*}{ Incontinencia urinaria } & \multirow{5}{*}{ Fuerte } & Micción inducida \\
\hline & & & Micción programada \\
\hline & & & Entrenamiento de la vejiga \\
\hline & & & Re-entrenamiento de los hábitos \\
\hline & & & Entrenamiento muscular pélvico \\
\hline & \multirow[t]{10}{*}{ Riesgo de caídas } & \multirow[t]{10}{*}{ Condicional } & $\begin{array}{l}\text { Programa de ejercicios multicomponente / entrenamiento } \\
\text { de la fuerza }\end{array}$ \\
\hline & & & $\begin{array}{l}\text { Evaluación del riesgo de caídas por el fisioterapeuta para } \\
\text { el desarrollo de prevención de caídas y lesiones }\end{array}$ \\
\hline & & & Ejercicios personalizados \\
\hline & & & Revisión de la medicación \\
\hline & & & Retirada de medicación psicotrópica \\
\hline & & & $\begin{array}{l}\text { Intervenciones multifactoriales con Evaluación Geriátrica } \\
\text { Integral }\end{array}$ \\
\hline & & & Modificación ambiental para la seguridad del hogar \\
\hline & & & $\begin{array}{l}\text { Tecnología de asistencia (ayuda para caminar, audífono, } \\
\text { sistema de alarma personal) }\end{array}$ \\
\hline & & & Evaluación del calzado \\
\hline & & & Inserción de un marcapasos \\
\hline \multirow{3}{*}{ Módulo III: Apoyo al cuidador } & \multirow{3}{*}{ Apoyo al cuidador } & \multirow{3}{*}{ Fuerte } & Cuidado del relevo \\
\hline & & & Apoyo psicosocial \\
\hline & & & Intervención de control activo \\
\hline
\end{tabular}

La OMS pretende que las pautas de esta guía ayuden a la implementación de la estrategia y plan de acción sobre Envejecimiento Saludable que se evaluarán con pruebas piloto en 20 países en 2020. Uno de estos países es Andorra, que colaborará en el marco del proyecto APTITUDE (Actuar para la Prevención Transpirenaica de la Dependencia de las personas mayores). Este proyecto tiene como meta implementar en la zona transfronteriza de los Pirineos, un modelo de prevención de la discapacidad en las personas mayores creando una red de promoción de la asistencia, la forma- ción, la investigación y la innovación en el ámbito de la gerontología (5).

Los lenguajes de enfermería estandarizados internacionalmente aceptados son: OMAHA System, NANDA-I (North American Nursing Diagnosis - International), NOC (Nursing Outcomes Classification), NIC (Nursing Interventions Clasifications), CIPE (International Classification of Nursing Practice), CCC (Clinical Care Classification) y ATIC (Architecture, Terminology, Interface-Information-Nursing and Knowledge) (6).

La taxonomía de la NANDA-I ofrece desde su fundación en 1982, un vocabulario enfermero estandarizado 
que permite poder clasificar los cuidados, estandarizarlos (posibilitando así el estudio de los mismos) y además dar apoyo a la toma de decisiones de las enfermeras para proveer una asistencia sanitaria de mayor calidad (7).

Gonzales y Santamaría (2018) exponen que el diagnóstico enfermero es el eje central del Proceso de Atención de Enfermería (PAE). Es la segunda fase del PAE siendo el resultado de la valoración para la planificación, ejecución y evaluación de la persona. Los profesionales de enfermería tras la valoración, identifican una o varias etiquetas diagnósticas que les permiten planificar las intervenciones y lograr los resultados parciales, totales o nulos del PAE (6).

Por consiguiente, la estandarización del lenguaje enfermero es perfectamente compatible con la individualización de los cuidados, en consonancia con un modelo enfermero y planificando los cuidados de la persona según su situación particular y de una forma holística. Posibilita el hecho de documentar los cuidados, el desarrollo del pensamiento crítico, la carga de trabajo de enfermería así como su inclusión en aplicaciones informáticas, estándares de información y registros electrónicos. El estudio de estos registros informáticos concede la toma de decisiones y la mejora del cuidado de las personas $(7,8)$.
Igualmente, la estandarización asegura la misma metodología de trabajo a las enfermeras, el acceso a la misma información para todos los profesionales y el intercambio de esta hacia los diferentes sistemas informáticos que soportan las Historias Clínicas Electrónicas a través de estándares como SNOMED CT (Systematized Nomenclature of Medicine Clinical Terms). Este se adapta a los requisitos de cada país que lo quiera implementar dentro de los sistemas informáticos sanitarios. Se utiliza para representar información clínicamente relevante de manera coherente, fiable y exhaustiva como parte integral de la producción y el intercambio de información electrónica (9).

Tal y como refieren Brito et al (2016), las personas con enfermedades crónicas son susceptibles de padecer numerosas necesidades de cuidados y servicios del sistema sanitario. Es por ello la importancia de poder cribar y hacer el seguimiento de las personas potencialmente frágiles y así enfocar el proceso de atención de manera efectiva (10).

Por lo citado anteriormente, se pretende estandarizar el diagnóstico de enfermería [00231] de NANDA-I: "Riesgo del Síndrome de la Fragilidad del Anciano" con los criterios de fragilidad de Fried y los de la GFST. Con la ayuda de 
ambos, se podrá realizar el seguimiento de las personas potencialmente frágiles por parte de Atención Primaria en Andorra en formato digital usando el estándar SNOMED CT de forma más eficiente $(9,11)$.

\section{MATERIAL Y MÉTODO}

Para abordar la estandarización del plan de cuidados del diagnóstico [00231] de NANDA-I "Riesgo del Síndrome de la Fragilidad del Anciano", se ha elegido la metodología Delphi en línea. Renguant Álvarez y Torrado Fonseca (2016) definen el método Delphi tradicional como la obtención de información apoyada en la consulta de grupos de expertos, de un área determinada, para conocer su criterio y juicio más fidedigno del grupo consultado.

Este método se basa en 4 particularidades: 1). Proceso iterativo, donde los expertos que participan dan su opinión o respuesta en más de una ocasión. Así tiene la oportunidad de reflexionar sus opiniones a través de cuestionarios y los resultados de los mismos. 2). Anonimato, donde los expertos no conocen la identidad de las respuestas de los otros expertos evitando así influencias y reactividades que puedan existir por diferencia de jerarquías de los expertos. 3). Retroalimentación o feedback controlado donde el grupo de investigación analiza las respuestas emitidas del problema que se aborda por el grupo de expertos y con estas establece la nueva consulta 4). La respuesta estadística del grupo donde se procesa la información de forma numérica con estimaciones de la mediana de las respuestas individuales para el resultado final del grupo. Este estudio tuvo en cuenta las 4 particularidades (12-13).

Según Varela et al (2012), el grupo coordinador oscila entre dos y cinco personas que estudian y afinan los siguientes aspectos: El protocolo de trabajo, la lista de los expertos, análisis de las rondas de respuesta y preparación de los siguientes cuestionarios, supervisar la retroalimentación y la marcha de todo el proceso así como la interpretación de los resultados finales (13).

Tras la definición del objetivo referido en la introducción, se procedió a la confirmación del grupo coordinador, donde se escogieron tres miembros del Grupo de Investigación en Ciencias de la Salud y Servicios Sanitarios de la Universidad de Andorra.

Una vez determinado el objeto de estudio y el grupo coordinador, el siguiente paso para llevar a cabo el Delphi se divide en 4 fases: I). Definición, donde se formula el objetivo para la consulta con el grupo de expertos. 2). Confirmación del grupo de informantes, donde se 
determina el perfil de los participantes y su ubicación, contacto con los integrantes potenciales, invitar y conseguir su compromiso de colaboración. El tamaño del grupo suele oscilar entre 7 y 30 en función del problema, aunque no es un condicionante, debe prevalecer siempre la calidad ante la cantidad. 3). Ejecución de las rondas de consulta, donde se elabora por un lado el cuestionario inicial, análisis de la información y confección de la siguiente ronda de feedback y por el otro lado, la consulta. Se ejecuta tantas veces como sea necesario para alcanzare el consenso que responda a los objetivos del estudio. Se deben categorizar y ordenar las respuestas en función del grado de acuerdo. El resultado será el punto de partida para las opiniones posteriores. En caso de que el experto difiera de la opinión general se le invitará a razonar sus respuestas. 4). Resultados, donde se analiza la información de la última ronda y elabora el informe de devolución final. El investigador podrá calcular el nivel de consenso para cada punto concreto, recoger las razones principales de disenso y finalmente calcular el nivel de relevancia $(14,15)$.

Los recientes avances tecnológicos han permitido avanzar hacía un Delphi en tiempo real o en línea, siendo una modificación del Delphi convencional en cuanto a reuniones "cara a cara" o de forma electrónica usando cuestionarios en línea (14).

El modelo de Fehring de 1994 mencionado por Cañon y Rodríguez (2010), para la validación de diagnósticos de enfermería consta de dos formas de validación: Por expertos y por validez clínica (16).

Escobar-Pérez y Cuervo Martínez (2008) definen el juicio de expertos como: "Una opinión informada de personas con trayectoria en el tema, que son reconocidas por otros como expertos cualificados en éste, y que pueden dar información, evidencia, juicios y valoraciones" (17).

La validación por expertos se mide mediante el modelo de "Validación del Contenido Diagnóstico" donde se consulta a enfermeras expertas. Fehring propone siete criterios para identificar en qué medida los expertos realmente lo son y se les asigna un peso donde se establece un mínimo de puntuación de cinco para ser considerado experto: 1). Doctorado en enfermería (4 puntos). 2). Doctorado en enfermería con el desarrollo de una tesis relevante para el diagnóstico de interés (1 punto). 3). Investigación publicada sobre el diagnóstico o contenido relevante por el mismo (2 puntos). 4). Artículo publicado del diagnóstico de interés en una revista científica con revisores (2 puntos). 5). Disertación 
oral de la tesis en diagnósticos (2 puntos). 6). Práctica clínica actual mínima de un año en un área relevante para el diagnóstico de interés (1 punto) y 7). Certificación en el área clínica relevante para el diagnóstico de interés (2 puntos) (16).

La filosofía de Patricia Benner (1984), citada por Brykczymski, en el libro de Raile Alligood. M (2015) y por Carrillo et al (2013), exhibe el procedimiento que la enfermera ejecuta desde la obtención de su graduado hasta que se especializa en un área asistencial determinada, realizando variaciones de conducta donde adquiere habilidades para una práctica profesional de mayor calidad $(18,19)$.

En este estudio, se ejecutaron las cuatro fases de la metodología Delphi. En la primera se definió el problema a resolver. Es decir, el objetivo del estudio; la estandarización del diagnóstico con los criterios de Fried y de la GFST para utilizarlo con SNOMED CT de modo más eficiente des de Atención Primaria.

Para poder estandarizar el plan de cuidados, se desarrolló un cuestionario con preguntas dirigidas en relación al diagnóstico, los resultados (NOC) y las intervenciones (NIC) relacionadas.

El diagnóstico [00231] de NANDA-I "Riesgo del Síndrome de la Fragilidad del Anciano" contiene cuarenta fac- tores de riesgo, veintiocho NOC y treinta y cuatro NIC. Se cogieron todos los NOC y todos los NIC para crear el cuestionario $(11,20,21)$

El objetivo del cuestionario fue escoger qué resultados y qué intervenciones incluirían los expertos del Delphi en los siete factores de riesgo seleccionados de los criterios de Fried y la GFST: 1). Agotamiento, 2). Alteración de la función cognitiva, 3). Caminar 4 metros en más de 5 segundos, 4). Disminución de la fuerza muscular, 5). Actividad física diaria media inferior a la recomendada según el sexo y la edad, 6). Pérdida involuntaria superior a $4,5 \mathrm{Kg}$ en un año y 7). Vivir solo.

Estos resultados e intervenciones incluidos en el cuestionario fueron los designados según los criterios de asociación de la herramienta informática NNNConsult de Elsevier, de la NANDA-I del 2015-2017.

En la segunda fase, se escogió la validación por expertos mediante el modelo de Fehring de "Validación de Contenido Diagnóstico" porque permite agrupar a los expertos más competentes en la temática de interés. Debido a la dificultad de la aplicación de los criterios del modelo de Fehring, se planteó la siguiente propuesta de adaptación del modelo, considerando la etapa de adqui- 
sición de habilidad de experto de la filo- sofía de Benner (tabla 2).

Tabla 2. Adaptación del sistema de puntuación de experto según el modelo de Fehring con la filosofía de Benner.

\begin{tabular}{|c|c|}
\hline Criterio (Fehring y Benner ) & Puntuación \\
\hline 1. Doctorado en enfermería. & 4 puntos \\
\hline $\begin{array}{l}\text { 2. Doctorado en enfermería con el desarrollo de una tesis relevante para el diagnóstico } \\
\text { enfermero de interés. }\end{array}$ & 1 punto \\
\hline 3. Investigación publicada sobre el diagnóstico o contenido relevante para el mismo. & 2 puntos \\
\hline 4. Artículo publicado en el diagnóstico en una revista científica con revisores. & 2 puntos \\
\hline $\begin{array}{l}\text { 5. Experta en la taxonomía NANDA-I (dominio, asimilación del "saber" práctico, visión } \\
\text { general y previsión de lo inesperado). }\end{array}$ & 2 puntos \\
\hline $\begin{array}{l}\text { 6. Experta en el área asistencial de la Fragilidad (dominio, asimilación del "saber" } \\
\text { práctico, visión general y previsión de lo inesperado). }\end{array}$ & 2 puntos \\
\hline
\end{tabular}

En la propuesta de adaptación se modificó el criterio 5 y 7 de Fehring para la etapa de adquisición de habilidades de experto de Benner entorno a la taxonomía de la NANDA-I y el área asistencial de la fragilidad. Por ello queda eliminado el criterio 6 ya que queda reflejado en el nuevo criterio de experto en fragilidad y se acepta el mismo valor numérico para cada criterio reemplazado (2 puntos) pudiendo así ponderar con la puntuación de Fehring, la inclusión de los expertos para el Delphi de la investigación. Al eliminarse un criterio de 1 punto, también se cambió el valor mínimo del modelo de Fehring de 5 a 4 puntos para la consideración de experto.

En la selección del grupo de expertos para el Delphi, se invitó a participar en el estudio a doce enfermeras expertas en cuanto a la adaptación del modelo de Fehring con la filosofía de Ben- ner en el contexto de la taxonomía de la NANDA-I y del área asistencial de la fragilidad con una puntuación mínima de 4 . Se garantizó el anonimato en sus respuestas en la encuesta en línea y en el formulario.

El contacto con ellos fue mediante un correo electrónico. En dicho correo se les explicó el objetivo de estudio, las instrucciones para poder responder al cuestionario en línea para la recopilación de datos con el enlace de acceso al cuestionario. Así mismo en el correo se adjuntó el consentimiento informado para su participación, que fue firmado y devuelto por todos los expertos que participaron.

En la tercera fase del Delphi, se creó un cuestionario en línea para ejecutar la primera ronda de consulta con el programa QuestionPro, donde se volvieron a exponer las instrucciones de res- 
puesta y el plazo límite de respuesta. Dicho cuestionario fue revisado por 6 expertos: 2 estudiantes de doctorado de la Universidad de Andorra (Grupo de Investigación Interdisciplinar en Educación), un doctor en de la Universidad de Andorra (especializado en el área de tecnología), una doctora en enfermería de la Universidad Blanquerna de Barcelona, una doctora de la Universidad de Barcelona y una enfermera especializada en lenguajes de enfermería de la NANDA-I.

Los expertos del Delphi, dispusieron de 30 días para proporcionar sus respuestas en la encuesta. Una vez obtenidas todas las respuestas del cuestionario en línea se realizó el análisis de las respuestas y de la ejecución de la segunda ronda de consulta creando un formulario. Este formulario fue creado con un Word con contraseña de acceso (se facilitó una distinta a cada uno de los expertos) y enviado por correo electrónico con las respuestas que no obtuvieron las ponderaciones de incluir o excluir del plan de cuidados, para consultar de nuevo con ellos si estas tenían que formar parte o no del plan de cuidados estandarizado. Para la respuesta del formulario los expertos tuvieron 15 días.

En la cuarta fase del Delphi se elaboró un informe con los resultados de los NOC y NIC escogidos para estandarizar el plan de cuidados con los factores de riesgo referidos.

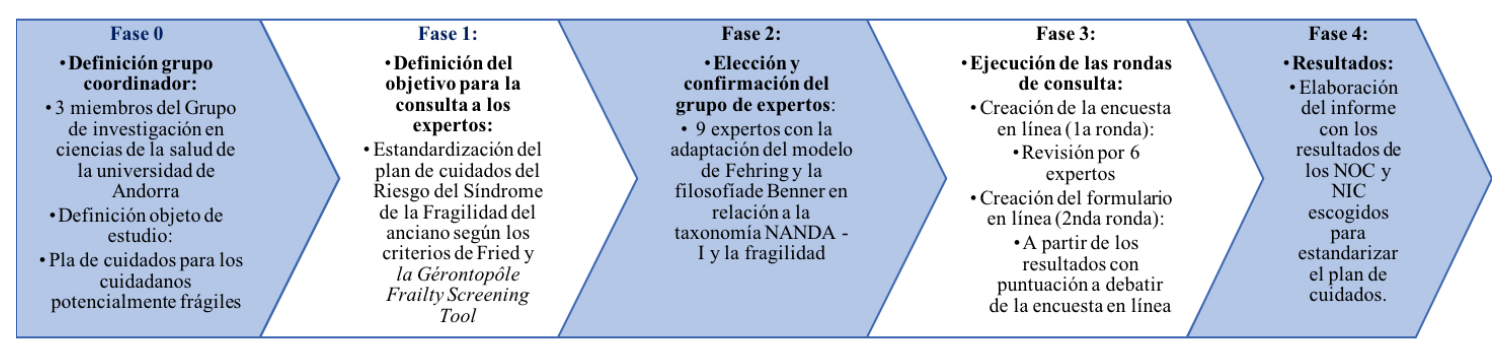

Figura 1. Adaptación del modelo Delphi convencional para la estandarización del plan de cuidados del Riesgo del síndrome de la fragilidad del anciano con los criterios de Fried y la Gérontopôle Frailty Screening Tool.

Referencia: Elaboración propia a partir del articulo "Reguant Álvarez M, Torrado Fonseca M. El método Delphi. REIRE, Rev d'Innovació i Recerca en Educació, 2016; 9 (1): 87-102. Disponible a: https://bit.ly/2AMZFMR" y de "Varela Ruiz M, Díaz Bravo L, García Durán R. Descripción y usos del método Delphi en investigación del área de la salud. Investig en Educ Médica, 2012; 1 (2): 90-95. Disponible a: https://bit.ly/2PnsdPv".

\section{REsultados}


La encuesta en línea fue enviada a doce expertos y se obtuvieron nueve respuestas representando un índex de participación del 75\%. Andorra dispone de once Centros de Salud, seis de estos nueve expertos pertenecen a seis Centros de Salud de Andorra y los otros tres expertos de dos Universidades de Enfermería de Catalunya.

Los resultados se calcularon haciendo una ponderación de las respuestas a partir de la escala Likert de seis puntos que tenia la encuesta para obtener los resultados.

La ponderación para las preguntas NOC y NIC fue dar el mismo valor que la escala de Likert a cada número de la columna de resultados. Se multiplicó el resultado de las respuestas con la ponderación y después se dividió por el número de respuestas.

Los intervalos de la ponderación se escogieron de forma empírica a partir de los resultados obtenidos. Estos fueron; si el resultado se encontraba entre el 1 y el 2.9 este quedaba excluido del plan de cuidados. Si se encontraba entre el 3 y el 4.4 se pediría la opinión al grupo de expertos Delphi para su inclusión o exclusión con el formulario. Si el resultado se encontraba entre 4,5 y 6 quedaba incluido (ver tabla 3).

Tabla 3. Ponderación de los resultados de los NOC y NIC a incluir, excluir o debatir con el grupo de expertos Delphi de la encuesta en línea y el formulario para la estandarización del plan de cuidados del diagnóstico del Riesgo del Síndrome de la Fragilidad del Anciano.

\begin{tabular}{c|c}
$\begin{array}{c}\text { Intervalos de ponderación en relación a la escalera } \\
\text { Likert de la encuesta en línea }\end{array}$ & $\begin{array}{c}\text { Plan de cuidados estandarizado del [00231] Riesgo } \\
\text { del síndrome de la fragilidad del anciano }\end{array}$ \\
\hline $\begin{array}{l}1 \text { y } 2.9 \\
3 \text { y } 4.5\end{array}$ & Excluir \\
\hline 4.5 y 6 & Debatir con grupo de expertos Delphi \\
\hline
\end{tabular}

Tabla 4. Resultado final de los NOC y NIC a incluir y excluir de la encuesta en línea y el formulario para la estandarización del plan de cuidados del diagnóstico del Riesgo del Síndrome de la fragilidad del anciano.

\begin{tabular}{|c|c|c|c|c|c|c|c|}
\hline \multirow{3}{*}{$\begin{array}{l}\text { NOC relacionado al } \\
\text { diagnóstico } \\
\text { principal según } \\
\text { NNNConsult }\end{array}$} & \multicolumn{7}{|c|}{$\begin{array}{c}\text { Factor de riesgo en relación a los criterios de Fried } \\
\text { y la Gérontopôle Frailty Screening Tool }\end{array}$} \\
\hline & \multicolumn{7}{|c|}{ NOC incluidos o excluidos del plan de curas estandarizado a partir de los resultados de la encuesta en línea } \\
\hline & Agotamiento & $\begin{array}{l}\text { Alteración de la } \\
\text { función cognitiva }\end{array}$ & $\begin{array}{l}\text { Caminar } 4 \\
\text { metros } \\
\text { requiere } \\
\text { más de } 5 \\
\text { segundos }\end{array}$ & $\begin{array}{l}\text { Disminución de } \\
\text { la fuerza } \\
\text { muscular }\end{array}$ & $\begin{array}{l}\text { Actividad física } \\
\text { diaria media } \\
\text { inferior a la } \\
\text { recomendada } \\
\text { según el sexo y } \\
\text { la edad. }\end{array}$ & $\begin{array}{l}\text { Perdida } \\
\text { involuntaria } \\
\text { superior a } \\
4,5 \mathrm{Kg} \text { en } \\
\text { un año. }\end{array}$ & Vivir solo \\
\hline $\begin{array}{l}\text { [3100] Autocontrol: } \\
\text { enfermedad aguda }\end{array}$ & NO & NO & NO & SI & NO & NO & NO \\
\hline $\begin{array}{l}\text { [3102] Autocontrol: } \\
\text { enfermedad crónica }\end{array}$ & NO & NO & NO & SI & $\mathrm{SI}$ & SI & SI \\
\hline
\end{tabular}


ene

De Migued-BARBERo RIESGo DE SÍndRome DE LA FRAgILIDAd deL Anciano

\begin{tabular}{|c|c|c|c|c|c|c|c|}
\hline $\begin{array}{l}\text { [2006] Estado de } \\
\text { salud personal }\end{array}$ & $\mathrm{SI}$ & SI & $\mathrm{SI}$ & SI & SI & SI & SI \\
\hline $\begin{array}{l}{[1302]} \\
\text { Afrontamiento de } \\
\text { problemas }\end{array}$ & NO & NO & NO & NO & NO & $\mathrm{SI}$ & $\mathrm{SI}$ \\
\hline [0200] Ambular & SI & NO & SI & SI & $\mathrm{SI}$ & NO & NO \\
\hline [1912] Caídas & $\mathrm{SI}$ & SI & $\mathrm{SI}$ & SI & SI & $\mathrm{SI}$ & $\mathrm{SI}$ \\
\hline $\begin{array}{l}\text { [0409] Coagulación } \\
\text { sanguínea }\end{array}$ & NO & NO & NO & NO & NO & NO & NO \\
\hline [0900] Cognición & SI & SI & NO & NO & NO & NO & $\mathrm{SI}$ \\
\hline $\begin{array}{l}\text { [1622] Conducta de } \\
\text { cumplimiento: dieta } \\
\text { prescrita }\end{array}$ & SI & SI & NO & SI & NO & SI & SI \\
\hline $\begin{array}{l}\text { [1909] Conducta de } \\
\text { Oprevención de } \\
\text { caídas }\end{array}$ & SI & SI & NO & SI & SI & SI & SI \\
\hline $\begin{array}{l}{[1804]} \\
\text { Conocimiento: } \\
\text { conservación de la } \\
\text { energía }\end{array}$ & SI & NO & SI & NO & SI & SI & NO \\
\hline $\begin{array}{l}\text { [1847] } \\
\text { Conocimiento: } \\
\text { manejo de la } \\
\text { enfermedad crónica }\end{array}$ & $\mathrm{SI}$ & SI & NO & NO & SI & SI & SI \\
\hline $\begin{array}{l}{[0204]} \\
\text { Consecuencias de } \\
\text { la inmovilidad: } \\
\text { fisiológicas }\end{array}$ & SI & NO & SI & SI & SI & SI & SI \\
\hline $\begin{array}{l}\text { [0205] } \\
\text { Consecuencias de } \\
\text { la inmovilidad: } \\
\text { psicocognitivas }\end{array}$ & NO & $\mathrm{SI}$ & NO & NO & $\mathrm{SI}$ & NO & SI \\
\hline $\begin{array}{l}{[0002]} \\
\text { Conservación de la } \\
\text { energía }\end{array}$ & SI & NO & $\mathrm{SI}$ & SI & SI & $\mathrm{SI}$ & NO \\
\hline [0202] Equilibrio & NO & NO & $\mathrm{SI}$ & SI & SI & NO & SI \\
\hline $\begin{array}{l}\text { [1004] Estado } \\
\text { nutricional }\end{array}$ & SI & SI & SI & SI & SI & SI & SI \\
\hline $\begin{array}{l}\text { [1008] Estado } \\
\text { nutricional: } \\
\text { ingestión } \\
\text { alimentaria y de } \\
\text { líquidos }\end{array}$ & $\mathrm{SI}$ & SI & NO & SI & $\mathrm{SI}$ & $\mathrm{SI}$ & $\mathrm{SI}$ \\
\hline $\begin{array}{l}\text { [2405] Función } \\
\text { sensorial }\end{array}$ & NO & SI & NO & NO & SI & NO & SI \\
\hline $\begin{array}{l}\text { [1503] Implicación } \\
\text { social }\end{array}$ & NO & SI & NO & NO & $\mathrm{SI}$ & NO & $\mathrm{SI}$ \\
\hline [0208] Movilidad & SI & NO & SI & SI & SI & SI & SI \\
\hline $\begin{array}{l}\text { [1211] Nivel de } \\
\text { ansiedad }\end{array}$ & NO & NO & NO & NO & NO & NO & NO \\
\hline $\begin{array}{l}\text { [0313] Nivel de } \\
\text { autocuidado }\end{array}$ & SI & SI & SI & SI & NO & SI & SI \\
\hline $\begin{array}{l}\text { [1208] Nivel de } \\
\text { depresión }\end{array}$ & NO & $\mathrm{SI}$ & NO & NO & NO & NO & SI \\
\hline $\begin{array}{l}\text { [0007] Nivel de } \\
\text { fatiga }\end{array}$ & SI & NO & SI & SI & $\mathrm{SI}$ & $\mathrm{SI}$ & NO \\
\hline $\begin{array}{l}\text { [1006] Peso: masa } \\
\text { corporal }\end{array}$ & NO & NO & $\mathrm{SI}$ & SI & NO & SI & SI \\
\hline $\begin{array}{l}\text { [1203] Severidad } \\
\text { de la soledad }\end{array}$ & NO & SI & NO & NO & NO & NO & SI \\
\hline $\begin{array}{l}\text { [1504] Soporte } \\
\text { social }\end{array}$ & SI & SI & NO & NO & SI & SI & SI \\
\hline
\end{tabular}




\begin{tabular}{|c|c|c|c|c|c|c|c|}
\hline $\begin{array}{l}\text { NIC relacionado al } \\
\text { diagnóstico principal }\end{array}$ & NIC incluido & el plan & andari & de los & la enc & & \\
\hline $\begin{array}{l}\text { [5602] Enseñanza: } \\
\text { proceso de } \\
\text { enfermedad }\end{array}$ & NO & NO & NO & NO & NO & NO & NO \\
\hline [6650] Vigilancia & SI & SI & $\mathrm{SI}$ & SI & SI & SI & SI \\
\hline $\begin{array}{l}{[5246]} \\
\text { Asesoramiento } \\
\text { nutricional }\end{array}$ & SI & NO & NO & SI & NO & SI & SI \\
\hline $\begin{array}{l}\text { [5440] Aumentar } \\
\text { los sistemas de } \\
\text { apoyo }\end{array}$ & SI & SI & NO & NO & SI & SI & SI \\
\hline $\begin{array}{l}\text { [1800] Ayuda con el } \\
\text { autocuidado }\end{array}$ & SI & SI & $\mathrm{SI}$ & SI & SI & SI & SI \\
\hline $\begin{array}{l}\text { [5330] Control del } \\
\text { estado de ánimo }\end{array}$ & SI & SI & $\mathrm{SI}$ & SI & SI & NO & SI \\
\hline $\begin{array}{l}\text { [5820] Disminución } \\
\text { de la ansiedad }\end{array}$ & NO & NO & NO & NO & NO & NO & SI \\
\hline $\begin{array}{l}\text { [5614] Enseñanza: } \\
\text { dieta prescrita }\end{array}$ & SI & NO & NO & SI & NO & SI & SI \\
\hline $\begin{array}{l}\text { [5606] Enseñanza: } \\
\text { individual }\end{array}$ & SI & SI & $\mathrm{SI}$ & SI & SI & SI & SI \\
\hline $\begin{array}{l}{[4760]} \\
\text { Entrenamiento de } \\
\text { la memoria }\end{array}$ & NO & SI & NO & NO & NO & NO & SI \\
\hline $\begin{array}{l}\text { [4720] Estimulación } \\
\text { cognitiva }\end{array}$ & NO & SI & NO & NO & SI & NO & SI \\
\hline $\begin{array}{l}{[8340] \text { Fomentar la }} \\
\text { resiliencia }\end{array}$ & NO & NO & NO & NO & SI & NO & NO \\
\hline $\begin{array}{l}\text { [0201] Fomento del } \\
\text { ejercicio: } \\
\text { entrenamiento de } \\
\text { fuerza }\end{array}$ & SI & NO & $\mathrm{SI}$ & SI & SI & $\mathrm{SI}$ & $\mathrm{SI}$ \\
\hline $\begin{array}{l}\text { [6480] Manejo } \\
\text { ambiental }\end{array}$ & SI & SI & $\mathrm{SI}$ & SI & SI & SI & SI \\
\hline $\begin{array}{l}\text { [6486] Manejo } \\
\text { ambiental: } \\
\text { seguridad }\end{array}$ & SI & SI & $\mathrm{SI}$ & SI & SI & NO & SI \\
\hline $\begin{array}{l}\text { [0180] Manejo de la } \\
\text { energía }\end{array}$ & SI & SI & $\mathrm{SI}$ & SI & $\mathrm{SI}$ & SI & SI \\
\hline $\begin{array}{l}\text { [3500] Manejo de } \\
\text { presiones }\end{array}$ & SI & NO & $\mathrm{SI}$ & SI & SI & NO & SI \\
\hline $\begin{array}{l}\text { [5230] Mejorar el } \\
\text { afrontamiento }\end{array}$ & SI & SI & NO & NO & SI & SI & SI \\
\hline $\begin{array}{l}\text { [4974] Mejorar la } \\
\text { comunicación: } \\
\text { déficit auditivo }\end{array}$ & NO & NO & NO & NO & SI & NO & SI \\
\hline $\begin{array}{l}\text { [4978] Mejorar la } \\
\text { comunicación: } \\
\text { déficit visual }\end{array}$ & NO & NO & NO & NO & SI & NO & SI \\
\hline $\begin{array}{l}\text { [4360] Modificación } \\
\text { de la conducta }\end{array}$ & NO & NO & NO & NO & NO & SI & $\mathrm{SI}$ \\
\hline $\begin{array}{l}{[1160]} \\
\text { Monitorización } \\
\text { nutricional }\end{array}$ & SI & SI & $\mathrm{SI}$ & SI & SI & SI & SI \\
\hline $\begin{array}{l}\text { [5100] Potenciación } \\
\text { de la socialización }\end{array}$ & SI & SI & NO & NO & SI & SI & SI \\
\hline $\begin{array}{l}\text { [6490] Prevención } \\
\text { de caídas }\end{array}$ & SI & SI & $\mathrm{SI}$ & SI & SI & SI & SI \\
\hline $\begin{array}{l}\text { [4010] Prevención } \\
\text { de hemorragias }\end{array}$ & NO & NO & NO & NO & NO & NO & NO \\
\hline
\end{tabular}




\begin{tabular}{|c|c|c|c|c|c|c|c|}
\hline $\begin{array}{l}\text { [3540] Prevención } \\
\text { de úlceras por } \\
\text { presión }\end{array}$ & SI & NO & $\mathrm{SI}$ & SI & SI & SI & $\mathrm{SI}$ \\
\hline $\begin{array}{l}\text { [0221] Terapia de } \\
\text { ejercicios: } \\
\text { ambulación }\end{array}$ & SI & NO & SI & SI & SI & NO & $\mathrm{SI}$ \\
\hline $\begin{array}{l}\text { [0226] Terapia de } \\
\text { ejercicios: control } \\
\text { muscular }\end{array}$ & SI & NO & SI & SI & SI & SI & SI \\
\hline $\begin{array}{l}\text { [0222] Terapia de } \\
\text { ejercicios: equilibrio }\end{array}$ & SI & NO & SI & SI & SI & SI & $\mathrm{SI}$ \\
\hline $\begin{array}{l}\text { [0224] Terapia de } \\
\text { ejercicios: } \\
\text { movilidad articular }\end{array}$ & SI & NO & SI & SI & SI & SI & $\mathrm{SI}$ \\
\hline $\begin{array}{l}\text { [5270] Apoyo } \\
\text { emocional }\end{array}$ & NO & $\mathrm{SI}$ & NO & NO & NO & NO & $\mathrm{SI}$ \\
\hline $\begin{array}{l}\text { [7110] Fomentar la } \\
\text { implicación familiar }\end{array}$ & SI & SI & NO & NO & SI & SI & SI \\
\hline $\begin{array}{l}{[6610]} \\
\text { Identificación de } \\
\text { riesgos }\end{array}$ & SI & SI & $\mathrm{SI}$ & SI & SI & SI & $\mathrm{SI}$ \\
\hline $\begin{array}{l}\text { [1100] Manejo de la } \\
\text { nutrición }\end{array}$ & SI & SI & SI & SI & SI & SI & SI \\
\hline
\end{tabular}

Como se menciono anteriormente, el diagnóstico [00231] de NANDA-I "Riesgo del Síndrome de la Fragilidad del Anciano" completo contiene cuarenta factores de riesgo, veintiocho NOC y treintaicuatro NIC. El resultado de la encuesta en línea y del formulario para la estandarización del plan de cuidados se muestra en la tabla 4.

En los resultados que aparecen en la tabla anteriormente nombrada, podemos apreciar el número de NOC y NIC que se incluye en los 7 factores de riesgo para la estandarización del plan de cuidados: 1). Agotamiento: Dieciséis NOC y veinticuatro NIC, descartando doce NOC y diez NIC. 2). Alteración de la función cognitiva: Quince NOC y diecisiete NIC, descartando dieciséis NOC y dieciséis NIC. 3). Caminar 4 metros en más de 5 segundos: Doce NOC y dieci- ocho NIC, descartando dieciséis NOC y dieciséis NIC. 4). Disminución de la fuerza muscular: Dieciséis NOC y veinte NIC, descartando doce NOC y doce NIC. 5). Actividad física diaria media inferior a la recomendada según el sexo y la edad: Dieciocho NOC y veintiséis NIC, descartando diez NOC y ocho NIC. 6). Pérdida involuntaria superior a $4,5 \mathrm{Kg}$ en un año: Diecisiete NOC y veintiún NIC, descartando once NOC y trece NIC y 7). Vivir solo: Veintiún NOC y treintaiún NIC, descartando siete NOC y tres NIC.

En el reciente articulo de Lucena et al (2019) podemos apreciar que se estudiaron propuestas de enlaces entre NOC, NIC para la práctica clínica de los diagnósticos de Riesgo y Síndrome de la Fragilidad del Anciano.

El estudio contó con la participación de 15 enfermeras especializadas 
del Departamento de Enfermería Gerontológica de Brasil, que respondieron a una encuesta en línea con la finalidad de escoger que 23 NOC y 20 NIC incluirían en el diagnostico de riesgo. Los NOC fueron escogidos por las autoras según los "Resultados principales para las especialidades de enfermería Gerontológica" y los NIC según las "Intervenciones básicas para áreas de especialidad de enfermería Gerontológica".

Los resultados NOC escogidos en el estudio para el riesgo, fueron 11 de los 23 iniciales: [0202] Equilibrio, [0900] Cognición, [1004] Estado nutricional, [3102] Autocontrol: enfermedad crónica, [1843] Conocimiento: manejo del dolor, [1633] Participación en el ejercicio, [1847] Conocimiento: manejo de la enfermedad crónica, [1828] Conocimiento: prevención de caídas, [1841] Conocimiento: manejo del peso, [2008] Estado de comodidad y [1808] Conocimiento: medicación.

Las intervenciones NIC escogidas en el estudio para el riesgo, fueron 7 de las 20 iniciales: [0200] Fomento del ejercicio, [5246] Asesoramiento nutricional, [2380] Manejo de la medicación, [4720] Estimulación cognitiva, [5100] Potenciación de la socialización, [5602] Enseñanza: proceso de enfermedad y [6490] Prevención de caídas (22).
Haciendo la comparativa de los dos estudios podemos observar que hay 5 resultados y 5 intervenciones que coinciden. Los cinco resultados son: 1). [0202] Equilibrio, [0900] Cognición, [1004] Estado nutricional, [3102] Autocontrol: enfermedad crónica y [1847] Conocimiento: manejo de la enfermedad crónica. Las cinco intervenciones son: [5246] Asesoramiento nutricional, [4720] Estimulación cognitiva, [5100] Potenciación de la socialización, [5602] Enseñanza: proceso de enfermedad y [6490] Prevención.

En el estudio de García-Mayor et al (2016) , se estudiaron 73 intervenciones con expertas de práctica avanzada para personas mayores en diferentes contextos asistenciales. En este estudio también podemos apreciar que coinciden las 5 intervenciones que en el estudio de Lucena (23).

\section{Discusión:}

El equipo de investigación de este estudio se decantó por el uso de la metodología Delphi porque permite resolver un problema de investigación a través de sus cuatro particularidades y sus cuatro fases. Se consideró que esta técnica era la que mejor resolvía el objetivo del estudio por la confirmación del panel de expertos, el proceso iterativo y la retroalimentación en cuanto a la se- 
lección de los NOC y NIC (con sus indicadores y intervenciones) que se podían representar en las 4 rondas de consulta a los expertos.

En el año 2001 Fried y otros autores observaron que varios ensayos clínicos habían mostrado que entre los elementos de intervención más prometedores para prevenir la fragilidad se encuentran la Atención Primaria y el seguimiento de las personas a largo plazo. Por otro lado, un obstáculo importante en el éxito de las intervenciones ha sido la ausencia de un método estandarizado y válido para el cribado y el seguimiento de las personas pre-frágiles y frágiles, para enfocarse de manera efectiva en la atención (3).

Se escogió el área asistencial de la fragilidad para realizar el estudio porque en primer lugar, como menciona Fried es una área donde la actuación de Atención Primaria juega un papel fundamental en su prevención y por la ausencia de un método estandarizado y validado para el seguimiento de las personas pre-frágiles.

En segundo lugar se consideró que era muy interesante para el aporte de conocimiento en la práctica clínica de la taxonomía NANDA-I referente a este diagnóstico, debido a la falta de estudios publicados entorno a la selección y validación de resultados e intervenciones como menciona Lucena et al (2019), así como a raíz de la colaboración de Andorra con la OMS en el proyecto APTITUDE que tiene en consideración las directrices de la guía ICOPE (5).

Esta guía nos expone a través de la amplia revisión bibliográfica efectuada por todos sus colaboradores, que la disminución de la $\mathrm{Cl}$ está frecuentemente caracterizada por problemas comunes en la edad avanzada como dificultades para oír, ver, recordar, moverse y realizar actividades diarias o sociales. Los primeros marcadores de disminución de la $\mathrm{Cl}$ son la disminución de la velocidad de la marcha y la disminución de la fuerza muscular, siendo a veces obviados por los profesionales sanitarios por falta de formación. Este hecho desencadena la necesidad de disponer de herramientas que les permitan identificar estos marcadores y así, prevenir o ralentizar la disminución de las $\mathrm{Cl}$.

Por un lado, los resultados de nuestro estudio podrían dar una herramienta a los profesionales de enfermería de Atención Primaria de Andorra en el seguimiento de las personas potencialmente frágiles en el proyecto APTITUDE. El plan de cuidados estandarizado con los 7 factores de riesgo (tabla 3 ) responde a todas las recomendaciones del módulo I y II de la guía ICOPE (tabla 1) 
permitiendo abordar la prevención y disminución de la $\mathrm{Cl}$. (5).

Por otro lado, el plan de cuidados estandarizado sí podría ser más eficiente en las consultas de Atención Primaria para los siete factores de riesgo porque solo se usarían los resultados y actividades más usadas según la opinión de los expertos, disminuyendo el número total y facilitando su uso por parte de los profesionales de enfermería en su cotidianidad.

\section{LIMITACIONES}

En el desarrollo de este estudio se han hallado dos limitaciones. La primera fue la muestra pequeña de expertos. Cabe destacar que estos disponían de un nivel de experto alto según la puntuación mayor de 4 puntos en el modelo de adaptación de Fehring con la filosofía de Benner.

La segunda limitación, fue realizar un cuestionario largo y extenso para poder determinar qué intervenciones y resultados se incluían o excluían del plan de cuidados. Se consideró necesario realizarlo así porque se quería preguntar en relación con los NOC y NIC del plan de cuidados completo para cada factor de riesgo con el objetivo de hacerlo más eficiente en su utilización diaria en la Atención Primaria. Este hecho desencadenó que se tuviera que ampliar el pe- ríodo de respuesta ya que tuvieron que contestar las preguntas en 7 encuestas, una para cada factor de riesgo haciendo largo el proceso de contestación.

\section{LÍNEAS FUTURAS}

Este estudio es la primera parte de nuestra investigación. La segunda parte se centrará en el mapeo con SNOMED CT del plan de cuidados estandarizado propuesto en esta investigación, para su posterior uso en una prueba de concepto por las enfermeras de Atención Primaria del Principado de Andorra. De esta forma podríamos intuir su estudio en una investigación de caso control a gran escala y de duración mínima de un año para validar la real eficacia de la investigación y la evolución del estado de salud de las personas potencialmente frágiles.

\section{CONCLUSIÓN}

Los elementos más prometedores para la prevención de la fragilidad son el cribado y el seguimiento des de Atención Primaria. Por consiguiente, esta investigación se está llevando a cabo con el fin de poder disponer de una herramienta digital que permita realizar el seguimiento de las personas potencialmente frágiles desde los Centros de Salud de Andorra. 
Con la intención de poder realizar el seguimiento, estimamos que disponiendo del plan de cuidados estandarizado en formato digital gracias al estándar SNOMED CT se podrá incluir a la Historia Clínica Electrónica de Andorra.

Consideramos que a través de los resultados de este estudio, la estandarización del diagnóstico [00231] de NANDA-I "Riesgo del Síndrome de la Fragilidad del Anciano" con los criterios de Fried y de la GFST permitirá realizar el seguimiento de forma más eficiente por parte de los profesionales de enfermería de los Centros de Salud de Andorra, ya que solo usarían según la opinión de los expertos los resultados y las intervenciones más relevantes para cada factor de riesgo.

\section{AGRADECIMIENTOS}

A todas las enfermeros/as que participaron de forma altruista como expertos en las dos rondas de consulta de este estudio, haciéndolo posible.

A la Sra. Carme Espinosa Fresnedo por transmitirme su entusiasmo, guía, estima y formación en esta taxonomía.

Al Dr. Aleix Dorca y la Dra. Betlem Sabrià por su imprescindible guía y ayuda en el cálculo de los resultados de esta investigación. 


\section{BIBLIOGRAFÍA}

1. Sist, X. (2016). Prevenció de la fragilitat en ancians pre-fràgils: efectivitat d'una intervenció a l'Atenció Primària. Tesis doctoral publicada. Universitat Autònoma de Barcelona, Barcelona.

2. Subra, J., Gillette-Guyonnet, S., Cesari, M., Oustric, S., y Vellas, B. (2012). Platform Team. The integration of frailty into clinical practice: Preliminary results from the Gérontopôle. J Nutr Health Aging,, 16, (8), 714-720. Consulta 1 de marzo, 2019, en: https://bit.ly/2rza36U

3. Fried, L.P., Tangen, C.M., Walston, J., Newman, A.B., Hirsch, C., Gottdiener, J., ... McBurnie, M.A. (2001). Frailty in Older Adults: Evidence for a Phenotype. The Journals of Gerontology: Series A, 56, (3), 146-157. Consulta 1 de marzo, 2019, en: https://bit.ly/349eq5Z

4. Subra, J., Gillette-Guyonnet, S., Cesari, M., Oustric, S., y Vellas, B. (2016). La integración de la fragilidad en la práctica clínica: resultados preliminares del estudio Gérontopôle en El libro blanco de la fragilidad. Hospital Universitario Gérontopôle de Toulose. Toulouse. 271-286. Consulta 1 de marzo, 2019, en : https://bit.ly/2opOdNX

5. World Health Organization. (2017). Integrated care for older people: guidelines on community-level interventions to manage declines in intrinsic capacity. Geneva: World Health Organization. Licencia: CC BY-NC-SA 3.0 IGO.

6. Gonzales. A, Santamaría García, JM. (2019). Los límites del lenguaje y su implicación sobre los límites del cuidado: a propósito de Wittgenstein y la enfermería. Ene enfermería. 12 (2): 1-20. Consulta 12 marzo, 2019, en: https://bit.ly/2F3w14W

7. Fernández Lasquetty - Blanc, B. (2012). Aplicación de Los Lenguajes Normalizados NANDA NOC NIC. Difusión Avances de Enfermería (DAE), Madrid: $2^{\mathrm{a}}$ edición

8. Lumillo Gutiérrez, I., Fabrellas Padrés, N., Ruymán Brito Brito, P., y Romero Sánchez, M. (2017). Influencia de los entornos de práctica en la actitud hacia el diagnóstico enfermero y su utilización. Ene enfermería, 11, (1). Consulta 12 marzo, 2019, en: https://bit.ly/2065uYM

9. Rius Soler, A., (2012, Mayo). Metodología de creación de subconjuntos de SNOMED CT. Foro Interoperabilidad. Comunicación presentada en la segunda reunión del Foro de Interoperabilidad de la terminología SNOMED CT, Segovia, España.

10.Brito Brito, PR., Sánchez Herrero, H., Ángel Fernandéz, D., García - Moreno, V., y RodríguezÁlvaro, M. (2016). Validación de contenido de la clasificación de diagnósticos enfermeros 2015-2017 de NANDA-I para el abordaje de la cronicidad en atención primaria. Ene enfermería, 10, (3). Consulta 12 marzo, 2019, en : https://bit.ly/ 2O51Le3
11.NANDA-I. (2015). "Riesgo del síndrome de la fragilidad del anciano en NANDA-I", en NANDA-I: Diagnósticos enfermeros: definiciones y clasificación 2015-2017. Elsevier, Barcelona, pp. 151.

12.Reguant Álvarez, M., y Torrado Fonseca, M. (2016). El método Delphi. REIRE, Rev d' Innovació i Recerca en Educació, 9, (1), 87-102. Consulta 5 marzo, 2019, en: http://revistes.ub.edu/index.php/ REIRE/article/view/reire2016.9.1916

13. Varela Ruiz, M., Díaz Bravo, L., y García Durán, R. (2012). Descripción y usos del método Delphi en investigación del área de la salud. Investig en Educ Médica, 1, (2), 90-95. Consulta 7 marzo, 2019, en : https://bit.ly/2PnsdPv

14.López Gómez, E. (2018). El método Delphi en la investigación actual en educación: una revisión teórica y metodológica. Educación XX1, 21(1), 1740. Consulta 7 marzo, 2019, en: https://bit.ly/2vn$\mathrm{z} 5 \mathrm{Vb}$

15.Cabero Almenara, J. (2014). Empleo del método Delphi y su empleo en la investigación en comunicación y educación. Edutec, 48, 1-16. Consulta 7 marzo, 2019, en: https://bit.ly/2G3tgTc

16.Cañon - Montañes, W., Rodríguez -Acelas, A. (2010). Desarrollo de la investigación en diagnósticos de enfermería. Revista Cuidarte, 1, (1), 7-8. Consulta 10 marzo, 2019, en: https://bit.ly/2O5d8CQ

17.Escobar-Pérez, J., Cuervo Martínez, A. (2008). Validez de contenido y juicio de expertos: una aproximación a su utilización. Avances en Medicina, 6, 27-36. Consulta el 11 noviembre, 2019, en: https://bit.ly/2NXPCrA

18.Carrillo Algara, A., García Serrano, L., Cárdenas Orujela, C., Díaz Sánchez, I. R., y Yaburdy Wilches, N. (2013). La filosofía de Patricia Benner y la práctica clínica. Enfermería Global. 32, 346-357. Consulta 10 marzo, 2019, en: https://bit.ly/2sbzANX

19. Brykczynski, K.A. (2015). "Capítulo 9, Patricia Benner. Filosofía del cuidado, sabiduría clínica y ética en la práctica de la enfermería" en Raile Alligood, M: Modelos y teorías en enfermería. Elsevier, Barcelona, pp. 118-25.

20.Moorhead, S., Swanson, E., Johnson, M., y Meridean, L.M. (2019). Clasificación de Resultados de Enfermería (NOC). Elsevier, Barcelona.

21.Bulechek, G.M., Butcher, H.K., Dochterman, J.M., y Wagner, C. (2018). Clasificación de Intervenciones de Enfermería (NIC). Elsevier, Barcelona.

22.Lucena. A, Argenta. C, Abreu Almeida. M, Moorhead. S y Swanson. E. (2019). Validation of Nursing Outcomes and Interventions to Older Adults Care with Risk or Frail Elderly Syndrome: Proposal of Linkages Among NOC, NIC and NANDA-I to clinical practice. International Journal of Nursing Knowledge, 30, (3), 147-153. Consulta el 20 noviembre, 2019, en: https://bit.ly/2rrS5n4 
23. Garcia-Mayor, S., Morilla- Herrera, J.C., Cuevas Fernandez - Gallego, M., Villa- Estrada, F., PorcelGalvez, A.M., Sastre- Fullana, P., y Morales - Ascencio, J.M (2016). Description of Advanced Practice Nurses Interventions Throught the Nursing
Interventions Classification in Different Care Setting for Older People: A Qualitative Study. International Journal of Nursing Knowledge, 27, (2), 79-86. Consulta el 20 noviembre, 2019, en: https:// bit.ly/36mroyO 